\title{
Potential of Local Hypovirulent Strains of Cryphonectria parasitica for Biological Control of Chestnut Blight
}

\author{
E. Gouveia, V. Coelho and L. Monteiro \\ IPB, University of Applied Sciences of Bragança \\ CIMO, Centro de Investigação de Montanha \\ Campus Santa Apolónia, 5301-037 Bragança \\ Portugal
}

Keywords: hypovirulence, Castanea sativa, biological control

\begin{abstract}
Cryphonectria parasitica, introduced in the NE of Portugal since 1989, is now an important and widespread pathogen of chestnut (Castanea sativa Mill.) which causes losses in chestnut fruit production and led to decline and death of many chestnut trees. Hypovirulence, a virus mediated attenuation of fungal pathogenesis, has not been frequent until now in Portugal but recently some cases of healing cankers were reported by chestnut growers. We assessed these sites and one of them, where healing cankers were clustered, was intensively surveyed. From all the trees with healing cankers, white and orange phenotypes of $C$. parasitica were isolated, which are both of the same vc type (EU-11). White isolates of $C$. parasitica showed reduced pigmentation in colony colour which was not completely stable, suppressed conidiation, reduction of phenol oxidase but no significant differences in lesion length and sporulation on chestnut twigs on dormant chestnut trees. Hypovirulent strains have dsRNA virus in the cytoplasm which is the typical element that reduces virulence in $C$. parasitica. Bands of dsRNA were identified in all of the white isolates and some variation in band dimension and number of bands was detected. The $L$ dsRNA (12 kbp), which is the diagnostic fragment of $C$. parasitica infection, was detected. The presence of white phenotypes of infected $C$. parasitica $(\mathrm{CHV})$ and the fact that spreads occur in natural conditions is promising for its development as a biological control agent of chestnut blight.
\end{abstract}

\section{INTRODUCTION}

Chestnut blight is an important and widespread disease of chestnut (Castanea sativa Mill.) in Portugal. Cryphonectria parasitica, the causal organism of the disease is classified as a quarantine organism, list A2, by the EPPO (OEPP/EPPO, 1982) and is also of quarantine significance for NAPPO and IAPSC. European chestnut is an important tree species in the ecological region of "Terra Fria” in the northeast of Portugal where more than $80 \%$ of the total area (28 $200 \mathrm{ha}$ ) of chestnut is found. Initial extensive surveys in that region, from 115 different sites in 20 different communities and around 10,000 inspected trees, had revealed 8-10\% of blighted trees with varied disease incidence among sites and communities (Gouveia et al., 2001). Recent surveys in areas where the disease was previously recognised have revealed an increase in disease incidence with $40-100 \%$ of infected trees (Bragança et al., 2007) which together with the large number of new infected sites greatly increase losses in chestnut fruit production and lead to decline and death of many chestnut trees.

The disease kills the infected tree branches and the rapid death of the entire tree takes place which is causing high environmental and economic concerns. Fungicides are not efficient in managing the disease and only canker excision and the removal of diseased branches are available in Portugal to reduce the dispersal of the disease. Despite the importance of these cultural measures to reduce the inoculum of the pathogen, the fragile chestnut ecosystem demands different disease management approaches. Biological control by hypovirulence, a virus mediated attenuation of fungal pathogenesis, is considered an efficient mean to control the disease and improve chestnut recovery (Heiniger and Rigling, 1994; Robin and Heiniger, 2001; Cortesi et al., 1989). 
Hypovirulent strains of $C$. parasitica are associated with the presence of an uncapsidated double stranded dsRNA virus in the cytoplasm of $C$. parasitica (Anagnostakis and Day, 1979; Dawe and Nuss, 2001). Hypovirus has no extracellular phase and virulent strains are converted to hypovirulent strains by the transmission of dsRNA through hyphal anastomoses between C. parasitica strains (Bissenger et al., 1997; Dawe and Nuss, 2001). Hypovirulence alters some morphological characteristics of the host fungi and hypovirulent strains show white colony morphology and reduced or absent conidial formation (Heiniger and Rigling, 1994; Dawe and Nuss, 2001).

Hypovirulence is not frequent in initial phases of the disease and ten or more years frequently elapse between blight arrival and hypovirulence appearance (Robin and Heiniger, 2001). In Portugal, after two decades of chestnut blight introduction, hypovirulence is not currently frequent. Despite the fact that some isolates have shown reduced levels of pigmentation and sporulation on PDA and some cankers seemed to be healing cankers, only one isolate of $C$. parasitica was identified until now in Portugal as $\mathrm{CHV}$ infected (Bragança et al., 2007). The population of C. parasitica in Portugal is characterized by general low vc type diversity (Bragança et al., 2007) and in the northeast many sub-populations have only one vc type (Gouveia et al., 2001). The dominant vc type is the EU-11, which is somewhat rare outside of Portugal, and is present only in some sites in Italy (Cortesi et al., 1996) and France (Robin and Heiniger, 2001). The success of hypovirulence depends on population structure related to vc and mating types (Heiniger and Rigling, 1994; Bissenger et al., 1997) however, as a therapeutic treatment the success of the hypovirulent strain depends on the vc type present in each individually treated canker.

Healing cankers in blighted chestnut trees are nowadays more frequently reported by chestnut growers in the NE of Portugal. The main objectives of this work were to isolate local hypovirulent strains of $C$. parasitica, to detect the presence of L-dsRNA, and to compare cultural characteristics, vegetative compatibility, phenol oxidase activity, hypovirulence conversion capacity and growth on dormant chestnut twigs to assess their potential for biological control of chestnut blight.

\section{MATERIALS AND METHODS}

In this study we focused on a chestnut orchard located in Vinhais in the northeastern region of Portugal where healing cankers of $C$. parasitica were reported. Chestnut trees were heavily blighted with extended and multiple cankers but some of them had visible healing cankers. The sampling was focused on the detection of hypovirulent strains of the pathogen. Individual samples of bark were collected from all the healing cankers, the surface was disinfected in $70 \%$ ethanol and promptly flamed. The samples were then placed on potato dextrose agar (PDAmb, $39 \mathrm{~g} \mathrm{~L}^{-1}$, Difco) supplemented with $1 \mathrm{mg} \mathrm{L}^{-1}$ of biotine and $100 \mathrm{mg} \mathrm{L}^{-1}$ of methionine. Plates were incubated at $25^{\circ} \mathrm{C}$ in the dark for seven days and then exposed to daylight for seven days. All the isolates were scored for the white mycelium and for the absence of picnydia and also scored for few and large pycnidia which are all indicators of infection by Cryphonectria hypovirus (CHV-1). One mycelial plug from each isolate was then transferred to fresh PDA.

All white $C$. parasitica isolates were chosen along with some random orange isolates to determine the vc-type using the method of Bissengger et al. (1997). Vegetative compatibility of the strains was assessed according to the barrage/merging response.

Conversion capacity was evaluated by pairing agar disks containing mycelia from white isolates and virulent isolates of known vc type on PDAmb, merging and spread of white colour were evaluated after 10 and 30 days.

Phenol oxidase activity test was performed by growing the isolates on modified Bavendamn's medium (Rigling et al., 1989) (0,5g taninic acid, 1,5\% Difco malt extract, $2 \%$ Difco bacto agar, $\mathrm{pH} 4,5$ adjusted with $1 \mathrm{~N} \mathrm{NaOH}$ ). Mycelial growth, in the three replications of each isolate, was measured after five and ten days of incubation at $25^{\circ} \mathrm{C}$. Changes in color were also checked. 
Growth on dormant chestnut twigs and sporulation capacity of the white isolates were assessed by inoculation of mycelial plugs on dormant twigs of chestnut trees as described by Elliston (1985). Inoculation points were protected with masking tape to prevent desiccation. Stems were incubated at room temperature and maintained in plastic containers. Lesion length and pycnospores production were evaluated after twenty days.

Extraction and detection of dsRNA was performed in selected $C$. parasitica isolates, that were phenotypically considered hypovirulent, according to the method of Morris and Dodds (1979) modified by Rigling et al. (1989) with some minor adaptations. Double stand of RNA was extracted from C. parasitica mycelium grown on cellophanecovered PDAmb. Mycelium was frozen in liquid nitrogen and ground with mortar and pestle. The tissue powder was mixed with $700 \mu \mathrm{l}$ of $2 \times \mathrm{STE}, 10 \%$ SDS (sodium dodecil sulfate) and the aqueous phase was transferred to $2 \mathrm{ml}$ Eppendorf tubes and vortexed with $700 \mathrm{\mu l}$ of phenol:chloroform:isoamylethanol (25:24:1) (Calbiochem). Following centrifugation (10 min, $5000 \mathrm{~g}$ ) the aqueous phase was recovered, extracted with the same volume of chloroform:isoamyl alcohol (24:1), vortexed and centrifugated again (10 min, $5000 \mathrm{~g}$ ). The aqueous phase was adjusted to $15 \%$ of ethanol and dsRNA was purified by chromatography on cellulose CF-11. The eluate from the column by adding (1×) STE was collected mixed with an equal volume of isopropanol and centrifuged at $4^{\circ} \mathrm{C}$ for $30 \mathrm{~min}$ at $16,000 \mathrm{rpm}$, washed with $70 \%$ ethanol and air dried. DsRNA was visualized in agarose gel stained with ethidium bromide.

\section{RESULTS}

\section{Healed Cankers and C. parastica Isolates}

All the trees of the chestnut orchard were blighted with extended and multiple cankers on their trunks and main stems. Six clustered trees revealed some healed or superficial cankers as well as aggressive cankers. All cankers in each diseased tree were sampled for $C$. parasitica isolation. Isolates of orange phenotype appeared in all cankers but in some cankers on different trees (VP2, VP3, VP5, VP6 and VP8) white phenotype was also detected. Orange isolates, grown in PDA medium, had a yellowish orange color and grew in an almost similar way. White phenotypes differed widely among them but were easily distinguished from orange isolates. Some of them remained white and some others developed a yellowish color and large pycnidia with scattered distribution on the colony. Successive replication stabilized colony morphology and the white color remained stable. Colonies with white and orange sectors were also frequent. When they were transferred to fresh PDA from the scarce pycnidia, some developed a singular phenotype with a white color and smooth center surrounded by whitish and orange sectors without pycnidia and with an aerial mycelium over the colony surface.

\section{Characteristics of White $C$. parasitica Isolates (vc-Type, Hypovirulence Conversion Capacity, Phenol Oxidase Activity and Lesion Length on Dormant Chestnut Twigs)}

Vegetative compatible tests have shown that all the isolates (white and orange) are of the same vc type identified as EU-11, the most common vc type at a regional scale.

The ability of white isolates to convert virulent isolates was assessed by pairing the white isolates with a known orange vc type isolate. Only individuals of the same vc type were converted and conversion was never observed when a different vc type was tested.

Phenol oxidase activity test performed in Bavendamn's medium has revealed a similar mycelial growth in all $C$. parasita isolates. White isolates always had a lower growth in Bavendamn's medium but differences were not statistically significant. Color reaction, which indicates phenol oxidase activity, was only evident after 10 days when white phenotypes maintained a light brown color, whereas virulent isolates acquired a dark brown color (Table 1).

All white $C$. parasitica isolates as well as virulent strains grew in dormant chestnut twigs. Both phenotypes had sporulated, and abundant pycnidia appeared in 
dormant chestnut twigs.

\section{Detection of dsRNA}

Electrophoretic patterns of dsRNA components from hypovirulent strains were found to be highly variable, with lengths falling into three broad size ranges L (large), $\mathrm{M}$ (medium) and S (small). M-dsRNA and S-dsRNA are internally deleted forms of L-dsRNA that persist during replication (Shapira et al., 1991). However, diagnosis of hypovirulence in $C$. parastica is confirmed by the detection of L-dsRNA. The great limitation for epidemiological study of dsRNA is the difficulty in detecting the dsRNA molecules. In our study, and because we did not include a positive control, band interpretation (including the absence of a band) was not conclusive and this limitation was solved when dsRNA was introduced as positive control and isolates with stable white characteristics were the ones selected to be tested for dsRNA. The initial studied colonies have shown a pattern of bands that revealed some dsRNA elements of $L$ and $M$ sizes and in some samples dsRNA of ribosomal origin. Detection of L-dsRNA was positive on $C$. parasitica isolates VP211, VP234, VP235, VP324, VP694 and VP811, which confirmed the presence of CHV on four of the six studied chestnut trees. Some positive L-dsRNA isolates (VP234, VP235) can be seen on Figure 1.

\section{DISCUSSION AND CONCLUSIONS}

European chestnut (Castanea sativa Mill.) assumes an important role for the environment, landscape and local economy, in the northeast of Portugal as a fruit extensive culture in a sustainable and characteristic agroforestry system. Chestnut blight has caused high chestnut tree mortality since its introduction and attempts for eradication of the disease by sanitary approaches have failed and sometimes worsened the dispersal of the disease by multiple and frequent cutting actions. Hypovirulence is a well known and specific biological control of chestnut blight (Grente, 1981) based on a dsRNA virus transmission (Anagnostakis and Day, 1979; Dawe and Nuss, 2001; Milgroom and Cortesi, 2004). Its specificity and efficacy are determined by biological traits related to vegetative compatibility (vc) of C. parasitica (Cortesi et al., 1989; Robin and Heiniger, 2001) in each specific site of application. Until recently, CHV was not detected in local populations of $C$. parasitica in Portugal. In this study we obtained and studied local white isolates of $C$. parasitica from healed and superficial cankers of chestnut blight. Generally, biological characteristics of our white $C$. parasitica isolates matched with European hypovirulent strains. Lesion length and sporulation on dormant chestnut twigs were unexpected results in the context of the virulence evaluation and results are being interpreted as saprophytic growth of hipovirulent strains, based on Prospero et al. (2006) and Gao and Shain (1995) that showed that a decreasing in turgidity in the bark promotes cankers expansion on dormant excised chestnut stems. Laboratorial and initial condition of chestnut stems may be similar to this situation and saprophytic growth and sporulation could be an ecological fitness for isolates that will promote dispersal and natural spread of hypovirulent strains.

Most hypovirulent strains can contain different number and concentration of multiple dsRNA elements (Anagnostakis and Day, 1979; Bissenger et al., 1997; Allemann et al., 1999; Montenegro et al., 2008). C. parasitica dsRNA elements are classified according to their estimated size in: L-dsRNA (ca $12 \mathrm{~kb}$ ), M-dsRNA (8-10 kb) and S-dsRNA (0,6-1,7 kb) (Shapira et al., 1991). In our work, multiple patterns appeared in the initial studied isolates, a commonly reported condition (Shapira et al., 1991; Dawe and Nuss, 2001). Detection of L-dsRNA is considered diagnostic for CHV infection and these dsRNA were detected on the white isolates VP211, VP234, VP235, VP324, VP694 and VP811.

Local conditions of $C$. parasitica population with one single vc type and the presence of perithecia that were observed at this time in old cankers and dead wood and consequently the probable existence of the two mating types of $C$. parasitica lead us to strongly advise the introduction of biological control based on local hipovirulent strains. 
A first experiment based on the local hypovirulent strain VP234 has already been implemented to obtain important data for a large scale application of this biological control method.

\section{ACKNOWLEDGEMENTS}

We thank the chestnut owners who contacted us to report hypovirulence on chestnut trees and Carlos Colinas of the Universitat of Lleida and Carlos Castanyo of the Centre Forestal Tecnológico de Catalunya for providing us with some hipovirulent strains of their collections.

\section{Literature Cited}

Allemann, C., Hoegger, P., Heiniger, U. and Rigling, D. 1999. Genetic of Cryphonectria hypoviruses (CHV1) in Europe assessed using restriction fragment length polymorphism (RFLP) markers. Molecular Ecology 8:843-845.

Anagnostakis, S. and Day, P. 1979. Hypovirulence conversion in Endothia parasitica. Phytopathology 69:1226-1229.

Bissenger, M., Rigling, D. and Heiniger, U. 1997. Population structure and disease development of Cryphonectria parasitica in European chestnut forests in the presence of natural hypovirulence. Phytophatology 87:50-59.

Bragança, H., Simões, S., Onofre, N., Tenreiro, R. and Rigling, D. 2007. Cryphonectria parasitica in Portugal: Diversity of vegetative compatibility type, mating type, and occurrence of hypovirulence. For. Path. 37:391-492.

Cortesi, P., Milgroom, M. and Bisiach, M. 1996. Distribution and diversity of vegetative compatibility in Cryphonectria parasítica. Mycological Research 100:1087-93.

Cortesi, P., Rigling, D. and Heiniger, U. 1989. Comparison of vegetative compatibility types Italian and Swiss subpopulations of Cryphonectria parasitica. Eur. J. For. Pathol. 28:167-176.

Dawe, A. and Nuss, D. 2001. Hypoviruses and chestnut blight: exploiting viruses to understand and modulate fungal pathogenesis. Ann. Rev. Genet. 35:1-29.

Elliston, J. 1985. Characteristics of dsRNA-containing strains of Endothia parasitica in relation to hipovirulence. Phytopathology 75:151-158.

Gao, S. and Shain, L. 1995. Effects of water stress on chestnut blight. Can. J. For. Res. 25:1030-1035.

Gouveia, E., Cardoso, P. and Monteiro, L. 2001. Incidence of chestnut blight and diversity of vegetative compatible types of Cryphonectria parasitica in Trás-osMontes. For. Snow Lands. Res. 76:387-390.

Grente, J. 1981. Les variants hypovirulents de Endothia parasitica, phénomène original en pathologie végétal.C. R. Acad. Sc. Paris 126(8):2347-2350.

Heiniger, U. and Rigling, D. 1994. Biological control of chestnut blight in Europe. Ann. Rev. Phytopathol. 32:581-99.

Milgroom, M. and Cortesi, P. 2004. Biological control of chestnut blight with hypovirulence: a critical analysis. Ann. Rev. Phytopathol. 42:311-38.

Montenegro, D., Aguin, O., Sainz, M., Hermida, M. and Mansilla, J. 2008. Diversity of vegetative compatible types, distribution of mating types and occurrence of hypovirulence of Cryphonectria paraitica in chestnut stands in NW Spain. Forest Ecology and Management 256:973-980.

Morris, J. and Dodds, J. 1979. Isolation and analysis of double-stranded RNA from virusinfected plant and fungal tissue. Phytopathology 69:854-858.

OEPP/EPPO. 1982. Data sheets on quarantine organisms. №69, Endothia parasitica. Bulletin OEPP/EPPO Bulletin 12(1).

Prospero, P., Conedera, M., Heiniger, U. and Rigling, D. 2006. Saprophytic activity and sporulation of Cryphonectria parasitica on dead chestnut wood in forest with naturally establised hypovirulence. Phytopathology 96(12):1337-1344.

Rigling, D., Heiniger, U. and Hohl, H. 1989. Reduction of laccase activity in dsRNAcontaining hypovirulent strains of Cryphonectria (Endothia) parasitica. 
Phytopathology 79:219-223.

Robin, C. and Heiniger, U. 2001. Chestnut blight in Europe: diversity of Cryphonectria parasitica, hypovirulence and biocontrol. For. Snow Lands. Res. 76:361-67.

Shapira, R., Choi, G. and Nuss, D. 1991. Virus-like genetic organization and expression strategy for a double-stranded RNA genetic element associated with biological control of chestnut blight. EMBO Journal 10:731-739.

\section{Tables}

Table 1. Mycelial growth and color reaction on Bavendamn's medium (phenol oxidase activity) after 10 days of white and orange isolates of Cryphonectria parasitica.

\begin{tabular}{lcc}
\hline C. parasitica strain & Mycelial growth & Color reaction \\
\hline VP211 & $3,90 \mathrm{a}$ & + \\
VP 234 & $3,56 \mathrm{a}$ & + \\
VP412 & $3,16 \mathrm{a}$ & + \\
VP511 & $2.56 \mathrm{a}$ & + \\
VP811 & $3,45 \mathrm{a}$ & + \\
PA228* & $3,46 \mathrm{a}$ & ++ \\
\hline ++ dark brown;+ light brown & & \\
*orange isolate & & \\
Means followed by the same letter are not significantly different according \\
to LSD multiple test, $\mathrm{P}<0,05$.
\end{tabular}

\section{Figures}

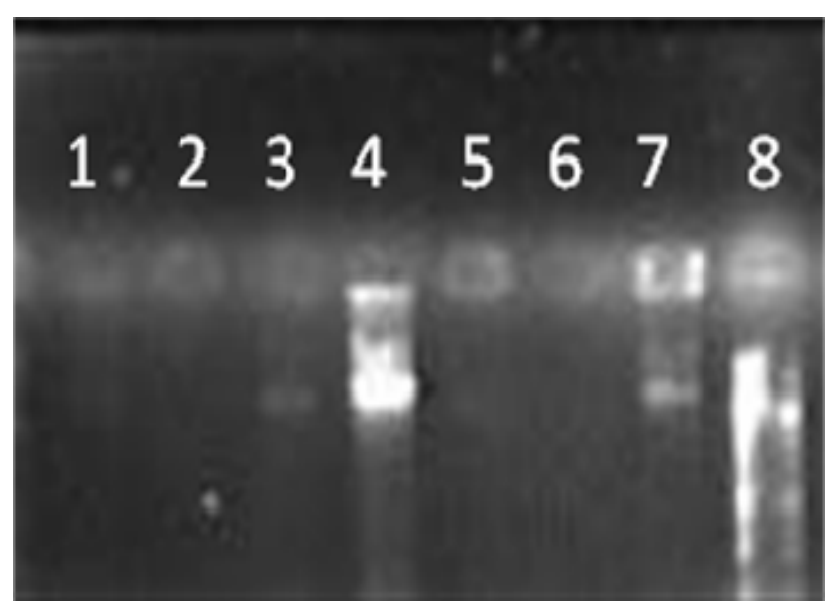

Fig. 1. Agarose gel electrophoresis of viral dsRNA molecules from Cryphonectria parasitica. Lane 1 - VP712, lane 2 - VP545, lane 3 - VP234, lane 4 - VP235, lane 5 - VP694, lane 6 - PA261(virulent strain), lane - 7 LL0559 (hypovirulent strain, positive reference), lane 8 - 1 kb DNA marker. 